\title{
ON CHARACTERIZING THE STANDARD QUANTUM LOGICS
}

BY

\author{
.W. JOHN WILBUR
}

\begin{abstract}
Let $\mathfrak{E}$ be a complete projective logic. Then $\mathcal{E}$ has a natural representation as the lattice of $\langle\cdot, \cdot\rangle$-closed subspaces of a left vector space $V$ over a division ring $D$, where $\langle\cdot, \cdot\rangle$ is a definite $\theta$-bilinear symmetric form on $V, \theta$ being some involutive antiautomorphism of $D$. Now a well-known theorem of Piron states that if $D$ is isomorphic to the real field, the complex field or the sfield of quaternions, if $\theta$ is continuous, and if the dimension of $\mathfrak{L}$ is properly restricted, then $\mathfrak{L}$ is just one of the standard Hilbert space logics.

Here we also assume $\mathfrak{L}$ is a complete projective logic. Then if every $\theta$-fixed element of $D$ is in the center of $D$ and can be written as $\pm d \theta(d)$, some $d \in D$, and if the dimension of $\mathcal{L}$ is properly restricted, we show that $\mathcal{E}$ is just one of the standard Hilbert space logics over the reals, the complexes, or the quaternions. One consequence is the extension of Piron's theorem to discontinuous $\theta$. Another is a purely lattice theoretic characterization of the lattice of closed subspaces of separable complex Hilbert space.
\end{abstract}

1. Introduction. A point of particular interest in the foundations of quantum theory is the apparently unique role of the logics based on classical Hilbert space. Efforts to account for this have focused largely on finding simple or physically plausible assumptions concerning a logic which will lead naturally to a characterization of the standard Hilbert space derived logics [18], [19], [14], [7], [8], [11], [12], [4]. This may be viewed as a two stage process. First, as suggested by the original work of Birkhoff and von Neumann [3], there are hypotheses relating the structure of the logic to the finite dimensional projective geometries. From this the coordinatization of the logic is deduced, i.e., the logic is projective. From this point assumptions must be found which relate the coordinatizing division ring $D$ to the field of real numbers. Zierler [18] assumes the compactness of certain sublattices of the

Presented to the Society, September 18, 1975; received by the editors April 20, 1976.

AMS (MOS) subject classifications (1970). Primary 06-00, 06A30, 46C05, 81A12; Secondary 06A25, 12J15, 15A63, 46K10, 50D99.

Key words and phrases. Complemented modular lattice, projective geometry, point, division ring, center of the ring, Archimedean field, non-Archimedean field, involutive antiautomorphism, $\theta$-bilinear symmetric form, complete projective logic, Hilbertian space, state, normalizable, probabilistic complete projective logic, infinite dimensional space, orthonormal sequence, standard quantum logics, separable Hilbert space.

- American Mathematical Society 1977 
logic together with a continuous nonconstant function from the unit interval to some finite dimensional sublattice of the logic, while Piron [14] simply postulates a rather direct relation between $R$ and $D$. More recently, Gudder and Piron [7], by assuming the existence of a smooth maximal observable, and Maczynski [12], by making assumptions on states and an observable, are able to deduce that $D$ is an extension of the real field $R$. If $D$ is a finite extension it follows that $D$ is either the real field, the complex field or the sfield of quaternions. We are principally concerned with this second stage. Our approach involves certain weak assumptions on $D$ and $\theta$, as set forth above, which must be satisfied if the elements of $V$ are to give rise to states on the logic in a natural manner as occurs with the standard logics. These hypotheses are discussed in $\$ 3$ while important implications in the general and infinite dimensional cases are derived in $\$ 4$ and $\$ 5$, respectively. As an application, in $\$ 6$ we present a system of axioms characterizing the lattice of closed subspaces of separable complex Hilbert space using only concepts common to lattice theory.

2. Preliminaries. Though we shall in general refer the reader to Varadarajan [15] for terminology and standard results concerning logics, projective geometries and orthocomplemented vector lattices, it seems appropriate to give here the definition of a projective logic, which is the central theme of our discussion. As given in [15] a logic $\mathcal{E}$ is projective if and only if it satisfies:

(i) given $a \neq 0$ in $\mathcal{L}$, there is a point $x<a$;

(ii) if $a \neq 0$ in $\mathcal{L}$ is the (lattice) sum of a finite set of points, then $\mathcal{L}[0, a]$ is a geometry of finite rank;

(iii) if $x, a \in \mathcal{L}, a \neq 0, \neq 1$ and $x$ is a point, then there are points $y$ and $z \in \mathcal{L}$ such that $y<a, z<a^{\perp}$ and $x<y \vee z$;

(iv) there exists at least one $a \in \mathcal{L}$ such that $4 \leqslant \operatorname{dim} \mathcal{L}[0, a]<\infty$.

The key to our discussion is the fact that complete projective logics have an equivalent formulation in terms of certain orthocomplemented vector lattices. Let $D$ be a division ring; $V$ a left vector space over $D$ with $4<\operatorname{dim} V ; \theta$ an involutive antiautomorphism of $D$; and $\langle\cdot, \cdot\rangle$ a definite, symmetric $(\theta(\langle x, y\rangle)=\langle y, x\rangle), \theta$-bilinear $(\langle a x, b y\rangle=a\langle x, y\rangle \theta(b)$ and distributive $)$ form on $V \times V$. If $M \subseteq V$ set

$$
M^{\perp}=\{x \in V:\langle x, y\rangle=0 \text {, for all } y \in M\} \text {. }
$$

Then a subspace $M$ of $V$ is called closed if $M^{\perp \perp}=M$; and the set $\mathcal{L}(V,\langle\cdot, \cdot\rangle)$ of all closed subspaces of $V$ under the natural inclusion forms a complete lattice with $\perp$ as an orthocomplementation. If, in addition, for each $M \in \mathcal{L}(V,\langle\cdot, \cdot\rangle), V=M+M^{\perp}$ algebraically, then $(V,\langle\cdot, \cdot\rangle)$ is said to be Hilbertian, and in this case $\mathcal{L}(V,\langle\cdot, \cdot\rangle)$ is a complete projective logic. In fact all complete projective logics are represented by such $\mathcal{L}(V,\langle\cdot, \cdot\rangle)$ with 
$(V,\langle\cdot, \cdot\rangle)$ Hilbertian. For the proof see $[15$, p. 179]. If $(V,\langle\cdot, \cdot\rangle)$ is Hilbertian we shall refer to $(V,\langle\cdot, \cdot\rangle, \theta, D)$ as a Hilbertian space. Thus there is an equivalence between the complete projective logics and the Hilbertian spaces.

Finally it is important to note that if $(V,\langle\cdot, \cdot\rangle, \theta, D)$ and $\left(V^{\prime}\right.$, $\left.\langle\cdot, \cdot\rangle^{\prime}, \theta^{\prime}, D^{\prime}\right)$ are two Hilbertian spaces which yield the same complete projective logic then $D$ and $D^{\prime}$ are isomorphic in such a way that $\theta$ and $\theta^{\prime}$ correspond naturally to each other while $\langle\cdot, \cdot\rangle$ carries over to the primed space to a multiple of $\langle\cdot, \cdot\rangle^{\prime}[15, \mathrm{pp} .33,59]$.

3. The probabilisitc hypotheses. A celebrated theorem of Gleason [6] asserts that all of the states on a standard logic arise naturally from normalized elements of any representing Hilbert space. While it is unknown whether this result also holds for the class of complete projective logics, yet if $(V$, $\langle\cdot, \cdot\rangle, \theta, D)$ is a Hilbertian space the elements of $V$ deserve consideration as a possible source of states on the accompanying logic. With this in mind we shall consider some conditions which a Hilbertian space must satisfy if each nonzero vector is to yield a state in the usual manner.

Let $(V,\langle\cdot, \cdot\rangle, \theta, D)$ be a Hilbertian space and let $x \in V$ with $x \neq 0$. Then foremost among our requirements would be the existence of a $d \in D$ with $\langle d x, d x\rangle= \pm 1$, i.e., $x$ must be normalizable. Clearly $\langle d x, d x\rangle= \pm 1$ if and only if $\langle x, x\rangle= \pm d^{-1} \theta\left(d^{-1}\right)$. Thus we might rephrase the normalizability condition as

$$
\text { for each } x \in V \text { there is a } d \in D \text { with }\langle x, x\rangle= \pm d \theta(d) \text {. }
$$

Now while the condition $(\dagger)$ is equivalent to the existence of normalizations for a given Hilbertian space, it is not entirely satisfactory because it can happen that one Hilbertian space may satisfy it while another Hilbertian space representing the same complete projective logic may not. An example may be constructed as follows. Let $A$ be a subfield of the real numbers in which $\sqrt{2}$ is never a sum of squares. Let $s$ be a sum of squares of elements in $A$. We claim $\sqrt{2}$ is again not a sum of squares in $A(\sqrt{s})$. If $\sqrt{s} \in A$, this is obvious; so suppose $\sqrt{s} \notin A$. Then each element of $A(\sqrt{s})$ has the form $a+b \sqrt{s}$ with $a, b \in A$. Suppose

$$
\sqrt{2}=\sum_{i=1}^{n}\left(a_{i}+b_{i} \sqrt{s}\right)^{2}
$$

Then rearranging terms yields

$$
\sqrt{2}=\left(\sum_{i=1}^{n} a_{i}^{2}+\left(\sum_{i=1}^{n} b_{i}^{2}\right) s\right)+\left(\sum_{i=1}^{n} 2 a_{i} b_{i}\right) \sqrt{s} .
$$

Clearly the coefficient of $\sqrt{s}$ in this equation must be nonzero or $\sqrt{2}$ would 
be a sum of squares in $A$. But then squaring both sides of the equation shows that $\sqrt{s} \in A$, a contradiction. Now starting with the field of rational numbers and repeatedly adjoining square roots of sums of squares we will arrive, after a transfinite process, at a field $A$ which has a square root for each sum of squares but cannot have a square root for $\sqrt{2}$ for that would make $\sqrt{2}$ a sum of squares. Let $V^{n}$ be a vector space of dimension $n>4$ over $A$ and let $\left\{e_{i}\right\}_{i=1}^{n}$ be a basis for $V^{n}$. Define $\langle\cdot, \cdot\rangle$ by $\left\langle e_{i}, e_{j}\right\rangle=\delta_{i j}$ and let $\theta$ be the identity isomorphism on $A$. Then $\left(V^{n},\langle\cdot, \cdot\rangle, \theta, A\right)$ is a Hilbertian space which satisfies $(\dagger)$. However $\left(V^{n},\langle\cdot, \cdot\rangle \sqrt{2}, \theta, A\right)$ is another Hilbertian space which yields the same complete projective logic but in which no element is normalizable. Thus in order that the existence of normalizations be an invariant of the underlying logic, and to avoid what seems a pathological situation, a slightly stronger assumption than ( $\dagger$ ) is necessary. Returning to the general Hilbertian space $(V,\langle\cdot, \cdot\rangle, \theta, D)$, let $F=\{d \in D: \theta(d)=$ $d\}$. Then though the details are elementary and best omitted, the weakest assumption which guarantees the condition ( $\dagger$ ) for all Hilbertian spaces with the same underlying logic as $(V,\langle\cdot, \cdot\rangle, \theta, D)$ is

$\left(\mathrm{P}_{1}\right) \quad$ for each $f \in F$ there is a $d \in F$ with $f= \pm d \theta(d)$.

If $C$ represents the center of $D$ we shall also impose on $(V,\langle\cdot, \cdot\rangle, \theta, D)$ the additional condition

$$
F \subseteq C \text {. }
$$

This condition is likewise independent of the representing Hilbertian space, i.e., an invariant of the logic. Its justification is that if $\langle x, x\rangle$ is to be a candidate for a probability then at least it should behave as a scalar and multiply commutatively in $D$. (Note that as one consequence of $P_{1}$, for each $f \in F$ there is an $x \in V$ with $f= \pm\langle x, x\rangle$.) We shall call a complete projective logic probabilisitc if and only if some representing Hilbertian space satisfies $P_{1}$ and $P_{2}$.

A condition which is sometimes easier or more convenient to apply than $P_{1}$ and $P_{2}$ is

$$
\text { given any } f \in F \text {, one of } f \text { or }-f \text { has a square root in } C \text {. }
$$

This is another invariant of the logic and is somewhat stronger than $P_{1}$ and $\mathrm{P}_{2}$. Let $(V,\langle\cdot, \cdot\rangle, \theta, D)$ be a Hilbertian space satisfying condition $\mathrm{N}$. Then clearly $\mathrm{P}_{2}$ holds. Now let $f \in F$ and suppose for $c \in C, f= \pm c^{2}$. Then $f=\theta(f)= \pm(\theta(c))^{2}$ and since $\theta(c)$ is also an element of the field $C$ we must have $\theta(c)= \pm c$. It follows that $f= \pm c \theta(c)$ and $P_{1}$ is established. We conclude that any complete projective logic represented by a Hilbertian space satisfying condition $\mathrm{N}$ is probabilistic. 
4. General results. Here we shall assume $(V,\langle\cdot, \cdot\rangle, \theta, D)$ is a Hilbertian space satisfying conditions $\mathrm{P}_{1}$ and $\mathrm{P}_{2}$ and shall present some results concerning the structure of $F$ and $D$.

First a remark on orientation. If for some $x \in V,\langle x, x\rangle=1$ we shall say $(V,\langle\cdot, \cdot\rangle, \theta, D)$ is positively oriented while if $\langle x, x\rangle=-1$ it will be called negatively oriented. To see that these categories are well defined, suppose $x, y \in V$ with $\langle x, x\rangle=1$ and $\langle y, y\rangle=-1$. Then if $M$ is the subspace of $V$ spanned by $x$ and $y, M \in \mathfrak{L}(V,\langle\cdot, \cdot\rangle)$ and since $V=M+M^{\perp}$ and $\operatorname{dim} V>4, M^{\perp} \neq\{0\}$. Let $z \in M^{\perp}$ and suppose $\langle z, z\rangle= \pm 1$. If $\langle z, z\rangle=$ 1 then $\langle y+z, y+z\rangle=0$ while if $\langle z, z\rangle=-1$ then $\langle x+z, x+z\rangle=0$. Either possibility contradicts the definiteness of $\langle\cdot, \cdot\rangle$. Throughout the remainder of our discussion we shall assume the positive orientation when convenient and only mention orientation when we feel it merits such attention.

4.1. THEOREM. The field $F$ has a natural ordering in which the set of positive elements is $P=\{\langle x, x\rangle: x \in V, x \neq 0\}$ if the space is positively oriented and is the negative of this set if the space is negatively oriented.

Proof. Since the positive and negative orientations differ by only a change in the sign of $\langle\cdot, \cdot\rangle$ it is sufficient to consider the positive orientation. Clearly $0 \notin P$. Let $f \in F$ and let $x \in V$ with $\langle x, x\rangle=1$. Then there is a $d \in D$ with $f= \pm d \theta(d)$, and it follows that $f= \pm\langle d x, d x\rangle$. We have shown that $P \cup\{0\} \cup-P=F$. Next let $a, b \in P$ with $a=\langle x, x\rangle$ for some $x \in V$. By $\mathrm{P}_{1}$ there is a $d \in D$ with $b=d \theta(d)$ (positive orientation), and by $\mathbf{P}_{2}$,

$$
a b=\langle x, x\rangle d \theta(d)=d\langle x, x\rangle \theta(d)=\langle d x, d x\rangle \in P .
$$

We have $P \cdot P \subseteq P$. Now let $z \in V$ with $\langle x, z\rangle=0$ and $\langle z, z\rangle=1$. Then

$$
a+b=\langle x+d z, x+d z\rangle \in P
$$

and $P+P \subseteq P$, and since $0 \notin P$ also, $P \cap-P=\varnothing$. This concludes the proof.

This result has some implications of interest. Eckman and Zabey [5] have shown that quantum mechanics is impossible (there are no orthocomplementations) in a Hilbert space over a finite or Galois field unless the Hilbert space is of dimension 2 and the characteristic of the field is not 2 . Their method is to enumerate the subspaces of various dimensions. Likewise Beltrametti and Cassinelli [1] have shown that quantum mechanics is impossible over any finite extension of a $p$-adic number field provided the dimension of the linear space is greater than or equal to five and the involutive antiautomorphism is assumed to be continuous. Here 4.1 shows that quantum mechanics is impossible in a space of dimension at least 4 (3 is actually sufficient for the argument, see [15, p. 61]) over any field of nonzero 
characteristic provided there are enough square roots to satisfy the hypothesis N. In particular, all algebraically closed fields of nonzero characteristic are included. That some such hypothesis concerning the algebraic nature of the field is necessary may be seen from the following example. Let $A$ be an arbitrary field and let $n \geqslant 4$ be a positive integer. Let $\left\{x_{1}, x_{2}, x_{3}, \ldots, x_{n}\right\}$ be a set of $n$ distinct indeterminants over $A$ and set $B=A\left(x_{1}, x_{2}, x_{3}, \ldots, x_{n}\right)$. Let $U$ be a vector space over $B$ of dimension $n$ with basis $\left\{e_{i}\right\}_{i=1}^{n}$. Then with $\theta=i_{B}$ and $\langle\cdot, \cdot\rangle$ defined by

$$
\left\langle\sum_{i=1}^{n} a_{i} e_{i}, \sum_{j=1}^{n} b_{j} e_{j}\right\rangle=\sum_{i=1}^{n} a_{i} b_{i} x_{i}
$$

on $U \times U,(U,\langle\cdot, \cdot\rangle, \theta, B)$ is a Hilbertian space.

4.2. THEOREM. The division ring $D$ has one of the following three forms:

(i) $D=F$;

(ii) $D=F(I)$ where $I^{2}$ is a negative element of $F$ in the natural order;

(iii) $D=F(I, J)$ where $I J=-J I$ and $I^{2}$ and $J^{2}$ are negative elements of $F$ in the natural order.

In case (ii) $\theta(I)=-I$ and in case (iii) $\theta(I)=-I$ and $\theta(J)=-J$.

Proof. Suppose $D \neq F$ and let $d$ be an element not in $F$. Then $d+\theta(d)$ $=f \in F$ and, since $F$ has characteristic zero, $f / 2 \in F$. Let $I=d-(f / 2)$. Then $\theta(I)=-I$ and hence $\theta\left(I^{2}\right)=I^{2} \in F$. Let $x \in V$ with $\langle x, x\rangle=1$. Then $\langle I x, I x\rangle=-I^{2}$ so $I^{2}$ is negative in the natural order of $F$. If $D=F(I)$ we have case (ii). Suppose $D \neq F(I)$. Let $e$ be any element not in $F(I)$. Then $e+\theta(e)=g \in F$ and, setting $J^{\prime}=e-(g / 2)$, we have, analogous to the result for $I, \theta\left(J^{\prime}\right)=-J^{\prime}$. Let $I J^{\prime}+\theta\left(I J^{\prime}\right)=a \in F$. Then $\theta\left(I J^{\prime}\right)=-I J^{\prime}+a=J^{\prime} I$. Set $J=J^{\prime}-\left(a / 2 I^{2}\right) I$. Then

$$
J I=J^{\prime} I-(a / 2)=-I J^{\prime}+(a / 2)=-I J .
$$

Since $\theta(J)=-J$ it follows as for $I$ that $J^{2}$ is negative in the natural order of $F$. If $D=F(I, J)$ we have case (iii). It remains to show $D$ cannot be larger than $F(I, J)$. Let $r$ be an element not in $F(I, J)$. Then $r+\theta(r)=h \in F$, and setting $P=r-(h / 2)$ we have $\theta(P)=-P$ and $P \notin F(I, J)$. Now set $I P+\theta(I P)=b \in F$ and $J P+\theta(J P)=c \in F$ and let $Q=P-\left(b / 2 I^{2}\right) I$ $-\left(c / 2 J^{2}\right) J$. Then a simple calculation shows that $I Q=-Q I$ and $J Q=$ - $Q J$. Again $Q \notin F(I, J)$. Now, however, $(I J) Q=Q(I J)$, and since

$$
\theta((I J) Q)=\theta(Q) \theta(I J)=(-Q)(-I J)=Q(I J),
$$

we have $(I J) Q \in F$ and hence $Q \in F(I, J)$. This contradiction completes the proof.

5. Infinite dimensional spaces and logics. The results of the previous section show that a complete projective logic which is probabilistic bears a close 
relation structurally to one of the classical logics over the reals, complexes, or quaternions. Here we shall investigate the consequences of the additional assumption that the logic is infinite dimensional.

In the case that $(V,\langle\cdot, \cdot\rangle, \theta, D)$ represents an infinite dimensional logic, $(V,\langle\cdot, \cdot\rangle, \theta, D)$ will be referred to as an infinite dimensional Hilbertian space. This is equivalent to the statement that $V$ is an infinite dimensional vector space over $D$.

Let $(V,\langle\cdot, \cdot\rangle, \theta, D)$ be an infinite dimensional Hilbertian space. Then it is possible, and convenient for our purposes, to give meaning to the notion of infinite sum. Let $\left\{u_{n}\right\}_{n=1}^{\infty}$ be an orthonormal sequence from $V$ (i.e., $\left\langle u_{n}, u_{m}\right\rangle$ $\left.=\delta_{n m}\right)$. Let $M$ be the subspace of $V$ spanned by $\left\{u_{n}\right\}_{n=1}^{\infty}$. Then if $x \in M^{\perp \perp}$ and $\left\langle x, u_{n}\right\rangle=b_{n}$ for $n=1,2, \ldots$, we shall write

$$
x=\sum_{n=1}^{\infty} b_{n} u_{n} .
$$

This gives a unique representation for the elements of $M^{\perp \perp}$ which agrees with the usual sum in the case when only finitely many of the $b_{n}$ 's are nonzero.

Before proceeding with our analysis of the infinite dimensional Hilbertian spaces we shall need a lemma concerning separable Hilbert space $\mathcal{H}$ over the real numbers $\mathbf{R}$. Elements of $\mathcal{H}$ will be written as infinite sequences $\left(\lambda_{i}\right)$ and $(\cdot, \cdot)$ will denote the inner product in $\mathcal{K}$. If $A$ is any subfield of $\mathbf{R}$ define $\mathcal{H}(A)$ by

$$
\mathscr{H}(A)=\left\{\left(\lambda_{i}\right) \in \mathscr{K} \mid \lambda_{i} \in A \text { for all } i\right\}
$$

Let $\mathcal{K}^{\circ}$ denote the finitely nonzero elements of $\mathcal{K}$ :

5.1. Lemma. Let $A$ be a subfield of $\mathbf{R}$ and let $x_{0}=\left(\lambda_{i}\right)$ and $y_{0}=\left(\mu_{i}\right)$ be orthogonal elements of $\mathcal{H}(A)$. Then there are sequences $\left\{x_{i}\right\}$ and $\left\{y_{i}\right\}$ such that

(i) $x_{i}, y_{i} \in \mathcal{H C}^{\circ} \cap \mathcal{H C}(A)$ for all $i, j>1$;

(ii) $x_{i} \perp y_{j}$ for all $i, j \geqslant 0$;

(iii) $x_{i} \rightarrow x_{0}$ and $y_{i} \rightarrow y_{0}$ in $\mathcal{H C}$.

Proof. Let $x_{1}=y_{1}=0$. Then we need only consider the recursion step. Thus we assume $\left\{x_{i}\right\}_{i=1}^{n}$ and $\left\{y_{i}\right\}_{i=1}^{n}$ have all been defined. Let $\left\{y_{i(p)}\right\}_{p=1}^{m}$ denote a maximal independent subsequence of $\left\{y_{i}\right\}_{i=1}^{n}$. Then set

$$
y_{i(p)}^{\prime}=y_{i(p)}-\sum_{q=1}^{p-1}\left(y_{i(p)}, y_{i(q)}^{\prime}\right)\left(y_{i(q)}^{\prime}, y_{i(q)}^{\prime}\right)^{-1} y_{i(q)}^{\prime} \quad \text { for } p=1,2,3, \ldots, m
$$

to define recursively the orthogonal set $\left\{y_{i(p)}^{\prime}\right\}_{p=1}^{m}$. Let

$$
z=y_{0}-\sum_{p=1}^{m}\left(y_{0}, y_{i(p)}^{\prime}\right)\left(y_{i(p)}^{\prime}, y_{i(p)}^{\prime}\right)^{-1} y_{i(p)}^{\prime}=\left(\nu_{i}\right) \text {. }
$$


Now choose $r$ large enough so that all $y_{i}, i<n$, are zero beyond the $r$ th place and

$$
\left|\sum_{i=1}^{r} \lambda_{i} \nu_{i}\right|<2^{-(n+1)}\left|\sum_{i=1}^{r} \nu_{i}^{2}\right|
$$

and

$$
\left|\sum_{i=r}^{\infty} \lambda_{i}^{2}\right|<2^{-(n+1)} .
$$

Let $x^{\prime}=\left(\lambda_{1}, \ldots, \lambda_{r}, 0,0,0, \ldots\right)$ and $z^{\prime}=\left(\nu_{1}, \ldots, \nu_{r}, 0,0,0, \ldots\right)$. Then set

$$
\begin{aligned}
x_{n+1} & =x^{\prime} \quad \text { if } z^{\prime}=0, \\
& =x^{\prime}-\left(x^{\prime}, z\right)\left(z^{\prime}, z^{\prime}\right)^{-1} z^{\prime} \text { otherwise. }
\end{aligned}
$$

Clearly $x_{n+1}$ has suitable coefficients and the right orthogonality properties. Also

$$
\left\|x-x_{n+1}\right\|<\left\|x-x^{\prime}\right\|+\left\|\left(x^{\prime}, z^{\prime}\right)\left(z^{\prime}, z^{\prime}\right)^{-1} z^{\prime}\right\|<2^{-(n+1)}+2^{-(n+1)}\left\|y_{0}\right\| .
$$

Now repeat the procedure beginning with $\left\{x_{i}\right\}_{i=1}^{n+1}$ and $\left\{y_{i}\right\}_{i=1}^{n}$ to obtain a $y_{n+1}$. This gives $\left\{x_{i}\right\}_{i=1}^{n+1}$ and $\left\{y_{i}\right\}_{i=1}^{n+1}$ with the desired properties and completes the recursion. Thus we have defined $\left\{x_{i}\right\}_{i=1}^{\infty}$ and $\left\{y_{i}\right\}_{i=1}^{\infty}$ which satisfy conditions (i)-(iii).

We shall now proceed to establish a kind of completeness property for infinite dimensional Hilbertian spaces satisfying $P_{1}$ and $P_{2}$. Actually a hypothesis somewhat weaker than $P_{1}$ and $P_{2}$ will suffice. We shall require:

The fixed set $F$ is an ordered subfield of $C$ and all the $\langle x, x\rangle, x \in V$, are nonnegative in $F$.

We shall use the term absolute value with its standard meaning in an ordered field. In accordance with the usage for non-Archimedean fields, an element of $F$ whose absolute value is less than any positive rational will be called infinitesimal while an element whose aboslute value is greater than any rational will be termed infinite.

5.2. LEMMA. Let $(V,\langle\cdot, \cdot\rangle, \theta, D)$ satisfy OR. Then for any $x, y \in V$ and any positive $\lambda \in F$,

$$
\langle x, y\rangle+\langle y, x\rangle \mid\left\langle\lambda^{-1}\langle x, x\rangle+\lambda\langle y, y\rangle\right. \text {. }
$$

Proof. If $\lambda$ is any positive element of $F, 0<\langle x-\lambda y, x-\lambda y\rangle$. Expanding, rearranging, and dividing by $\lambda$ gives

$$
\langle x, y\rangle+\langle y, x\rangle\left\langle\lambda^{-1}\langle x, x\rangle+\lambda\langle y, y\rangle .\right.
$$


Similar treatment of $0<\langle x+\lambda y, x+\lambda y\rangle$ then yields the result.

5.3. Lemma. Let $(V,\langle\cdot, \cdot\rangle, \theta, D)$ be an infinite dimensional Hilbertian space satisfying OR. Let $A$ be a subfield of $F$ which is order isomorphic to a naturally ordered subfield of the real numbers and let $\mathbf{R}$ denote an extension of $A$ to the real numbers. If $\left\{a_{i}\right\}_{i=1}^{\infty}$ is a sequence from $A$ with $a_{1} \neq 0$ and which is square summable in $\mathbf{R}$ to an element of $A$ and if $\left\{u_{i}\right\}_{i=1}^{\infty}$ is an orthonormal sequence in $V$, then there is an $x=\sum_{i=1}^{\infty} b_{i} u_{i} \in V$ which satisfies:

(i) $\left(a_{k}-b_{k}\right) \theta\left(a_{k}-b_{k}\right)$ is infinitesimal for all $k$;

(ii) $\langle x, x\rangle-\sum_{i=1}^{\infty} a_{i}^{2}$ is infinitesimal.

Proof. Let $\mathcal{X}$ denote separable Hilbert space over $\mathbf{R}$ and let $p=\left(a_{i}\right)$ and $q=(1,0,0,0, \ldots)$ in $\mathcal{H}$. Then set

$$
p^{\prime}=(p, p)(q, p)^{-1} q-p
$$

and

$$
q^{\prime}=(p, p)(q, p)^{-1} q=\left((p, p)(q, p)^{-1}, 0,0,0, \ldots\right) .
$$

We have $q^{\prime}=p+p^{\prime}$ and $\left(p, p^{\prime}\right)=0$. Applying 5.1 to $p$ and $p^{\prime}$ in $\mathcal{H}$ we obtain sequences $\left\{p_{i}\right\}$ and $\left\{p_{i}^{\prime}\right\}$ from $\mathcal{H}^{\circ} \cap \mathcal{H C}(A)$ converging to $p$ and $p^{\prime}$, respectively. Now each element $\left(\lambda_{i}\right)$ of $\mathcal{F}^{0} \cap \mathcal{H}(A)$ corresponds to an element $\sum_{i=1}^{\infty} \lambda_{i} u_{i}$ of $V$ and $(\cdot, \cdot)$ acts on the first just as $\langle\cdot, \cdot\rangle$ does on the second. Let $\left\{r_{i}\right\}$ and $\left\{r_{i}^{\prime}\right\}$ denote the sequences in $V$ corresponding to the sequences $\left\{p_{i}\right\}$ and $\left\{p_{i}^{\prime}\right\}$ in $\mathcal{H}^{\circ} \cap \mathcal{H}(A)$. Now let $\rho$ be a positive rational element of $A$ and choose $n$ large enough so that $\left\|p-p_{n}\right\|<\rho$ and $\left\|p^{\prime}-p_{n}^{\prime}\right\|$ $<\rho$. Then $\left(q^{\prime}-p_{n}-p_{n}^{\prime}, q^{\prime}-p_{n}-p_{n}^{\prime}\right)<(2 \rho)^{2}$ and with $z=(p, p)(q, p)^{-1} u_{1}$ we have $\left\langle z-r_{n}-r_{n}^{\prime}, z-r_{n}-r_{n}^{\prime}\right\rangle\left\langle(2 \rho)^{2}\right.$. Let $M$ be the subspace of $V$ generated by $\left\{r_{i}\right\}_{i=1}^{\infty}$. Then we may write $z=x+y$ with $x \in M^{\perp \perp}$ and $y \in M^{\perp}$. It follows that $\left\langle x-r_{n}, x-r_{n}\right\rangle<(2 \rho)^{2}$. If $M_{1}$ is the subspace of $V$ generated by $\left\{u_{i}\right\}_{i=1}^{\infty}$, then $M \subset M_{1}$ and, hence, $x \in M^{\perp \perp} \subset M_{1}^{\perp \perp}$. Thus $x=\sum_{i=1}^{\infty} b_{i} u_{i}$ for a proper choice of $b_{i} \in D$. We must show that $x$ and $p$ satisfy conditions (i) and (ii).

We begin with relation (i). Let $k$ be a fixed integer. Let $p_{n}=\left(c_{i}\right)$. Then $r_{n}=\sum_{i=1}^{\infty} c_{i} u_{i}$. Now if $s$ is the projection of $p-p_{n}$ onto the $k$ th coordinate space then because $\left\|p-p_{n}\right\|<\rho$ we have $(s, s)<\rho^{2}$. Translating into $V, s$ corresponds to $a_{k} u_{k}-c_{k} u_{k}$ yielding $\left\langle a_{k} u_{k}-c_{k} u_{k}, a_{k} u_{k}-c_{k} u_{k}\right\rangle\left\langle\rho^{2}\right.$. Also the projection of $x-r_{n}$ onto the $k$ th coordinate space is $b_{k} u_{k}-c_{k} u_{k}$ and by virtue of $\left\langle x-r_{n}, x-r_{n}\right\rangle\left\langle(2 \rho)^{2}\right.$ we have $\left\langle b_{k} u_{k}-c_{k} u_{k}, b_{k} u_{k}-c_{k} u_{k}\right\rangle<$ $(2 \rho)^{2}$. Now applying 5.2 we obtain 


$$
\begin{aligned}
\left\langle a_{k} u_{k}-\right. & \left.b_{k} u_{k}, a_{k} u_{k}-b_{k} u_{k}\right\rangle \\
= & \left\langle\left(a_{k} u_{k}-c_{k} u_{k}\right)+\left(c_{k} u_{k}-b_{k} u_{k}\right),\left(a_{k} u_{k}-c_{k} u_{k}\right)+\left(c_{k} u_{k}-b_{k} u_{k}\right)\right\rangle \\
\langle & \rho^{2}+(2 \rho)^{2}+\left\langle a_{k} u_{k}-c_{k} u_{k}, c_{k} u_{k}-b_{k} u_{k}\right\rangle \\
& \quad+\left\langle c_{k} u_{k}-b_{k} u_{k}, a_{k} u_{k}-c_{k} u_{k}\right\rangle \\
< & 5 \rho^{2}+\rho^{-1}\left(\rho^{2}\right)+\rho(2 \rho)^{2}=\rho+5 \rho^{2}+4 \rho^{3} .
\end{aligned}
$$

Since by choosing $n$ large enough $\rho$ can be as small a rational as desired, (i) follows.

We move to the consideration of (ii). We must first establish an upper bound for $\left\langle r_{n}, r_{n}\right\rangle$. By a series of rearrangements and an application of 5.2,

$$
\begin{aligned}
\left\langle x-r_{n}, x-r_{n}\right\rangle\left\langle 4 \rho^{2},\right. \\
\langle x, x\rangle-\left\langle x, r_{n}\right\rangle-\left\langle r_{n}, x\right\rangle+\left\langle r_{n}, r_{n}\right\rangle\left\langle 4 \rho^{2},\right. \\
\left\langle r_{n}, r_{n}\right\rangle\left\langle 4 \rho^{2}-\langle x, x\rangle+\left(\left\langle r_{n}, x\right\rangle+\left\langle x, r_{n}\right\rangle\right)\right. \\
\left\langle 4 \rho^{2}-\langle x, x\rangle+2\langle x, x\rangle+2^{-1}\left\langle r_{n}, r_{n}\right\rangle,\right. \\
\left\langle r_{n}, r_{n}\right\rangle\left\langle 8 \rho^{2}+2\langle x, x\rangle .\right.
\end{aligned}
$$

Employing this result and another application of 5.2 we have

$$
\begin{gathered}
\left\langle x-r_{n}, x-r_{n}\right\rangle\left\langle 4 \rho^{2},\right. \\
\langle x, x\rangle-\left\langle r_{n}, x\right\rangle-\left\langle x, r_{n}\right\rangle+\left\langle r_{n}, r_{n}\right\rangle\left\langle 4 \rho^{2},\right. \\
\langle x, x\rangle-\left\langle r_{n}, r_{n}\right\rangle\left\langle 4 \rho^{2}+\left\langle r_{n}, x\right\rangle+\left\langle x, r_{n}\right\rangle-2\left\langle r_{n}, r_{n}\right\rangle,\right. \\
\langle x, x\rangle-\left\langle r_{n}, r_{n}\right\rangle\left\langle 4 \rho^{2}+\left\langle r_{n}, x-r_{n}\right\rangle+\left\langle x-r_{n}, r_{n}\right\rangle\right. \\
\left\langle 4 \rho^{2}+\rho^{-1}\left\langle x-r_{n}, x-r_{n}\right\rangle+\rho\left\langle r_{n}, r_{n}\right\rangle\right. \\
\left\langle 4 \rho^{2}+4 \rho+8 \rho^{3}+2 \rho\langle x, x\rangle .\right.
\end{gathered}
$$

Starting with the same initial inequality and using similar rearrangements we may arrive at

$$
\left\langle r_{n}, r_{n}\right\rangle-\langle x, x\rangle\left\langle 4 \rho^{2}+4 \rho+\rho\langle x, x\rangle\right.
$$

Now $\langle x, x\rangle\langle\langle z, z\rangle$ and hence $\langle x, x\rangle$ is finite. It follows that for any preassigned rational $\varepsilon>0$, for all $n$ large enough, $\left|\langle x, x\rangle-\left\langle r_{n}, r_{n}\right\rangle\right|<\varepsilon$. By virtually identical arguments in $\mathscr{H}$ we also have $\left|(p, p)-\left(p_{n}, p_{n}\right)\right|<\varepsilon$ for all $n$ large enough. Thus for all $n$ large enough $|\langle x, x\rangle-(p, p)|\langle 2 \varepsilon$. Since this last expression does not depend on $n$ the result (ii) follows.

While this lemma will prove useful in the non-Archimedean case, our first application will be a completeness result on Archimedean fields. Somewhat similar results in a different setting have been obtained by Morash [13] and Holland [9]. 
5.4. THEOREM. Let $(V,\langle\cdot, \cdot\rangle, \theta, D)$ be an infinite dimensional Hilbertian space satisfying OR. If there is an orthonormal sequence $\left\{u_{i}\right\}_{i=1}^{\infty}$ in $V$ and if $F$ is Archimedean, then $F$ is isomorphic to the real numbers and $(V,\langle\cdot, \cdot\rangle, \theta, D)$ satisfies $\mathbf{N}$.

Proof. It is only necessary to show that $F$ is isomorphic to the real numbers because $\mathrm{N}$ is then an obvious consequence. Let $F=A$ in 5.3 and let $r \in \mathbf{R}-A$ where $r>0$. Let $q$ be a rational number with $0<r<q$. Then a sequence of positive rationals $\left\{a_{i}\right\}_{i=1}^{\infty}$ may be found satisfying $\sum_{i=1}^{\infty} a_{2 i}^{2}=r$ and $\sum_{i=1}^{\infty} a_{2 i-1}^{2}=q-r$. Since infinitesimal means zero in $F, 5.3$ yields the existence of $x=\sum_{i=1}^{\infty} a_{i} u_{i} \in V$ with $\langle x, x\rangle=q$. Let $M$ be the subspace of $V$ generated by $\left\{u_{2 i}\right\}_{i=1}^{\infty}$. Then we may write $x=s+t, s \in M^{\perp \perp}$ and $t \in$ $M^{\perp}$. Clearly $s=\sum_{i=1}^{\infty} a_{2 i} u_{2 i}$ and $t=\sum_{i=1}^{\infty} a_{2 i-1} u_{2 i-1}$. Now if for each $n$, $s_{n}=\sum_{i=1}^{n} a_{2 i} u_{2 i}$ then

$$
\sum_{i=1}^{n} a_{2 i}^{2}=\left\langle s_{n}, s_{n}\right\rangle \leqslant\langle s, s\rangle
$$

for each $n$. It follows that $r \leqslant\langle s, s\rangle$. Likewise $q-r \leqslant\langle t, t\rangle$. Since $\langle s, s\rangle+$ $\langle t, t\rangle=\langle x, x\rangle=q$, necessarily $r=\langle s, s\rangle$ and $r \in F$. This contradiction establishes the result.

We return now to a consideration of the probabilistic logics. We shall need several lemmas before we reach our main theorem.

5.5. Lemma. Let $(V,\langle\cdot, \cdot\rangle, \theta, D)$ be an infinite dimensional Hilbertian space satisfying $\mathrm{P}_{1}$ and $\mathrm{P}_{2}$. Let $\left\{u_{i}\right\}_{i=1}^{\infty}$ and $\left\{v_{i}\right\}_{i=1}^{\infty}$ be orthonormal sequences in $V$ which are orthogonal to each other. Then if $x=\sum_{i=1}^{\infty} a_{i} u_{i} \in V$, we also have $x^{\prime}=\sum_{i=1}^{\infty} a_{i} v_{i} \in V$ and $\left\langle x^{\prime}, x^{\prime}\right\rangle=\langle x, x\rangle$.

Proof. Since $2^{-1}$ is positive in $F$ there is by $\mathrm{P}_{1}$ on $\alpha \in D$ with $\alpha \theta(\alpha)=$ $2^{-1}$. Now let $M_{1}$ and $M_{2}$ be the subspaces generated by the orthonormal sequences $\left\{\alpha u_{i}+\alpha v_{i}\right\}_{i=1}^{\infty}$ and $\left\{\alpha u_{i}-\alpha v_{i}\right\}_{i=1}^{\infty}$, respectively. Then we may write $x=r_{1}+r_{2}, r_{1} \in M_{1}^{\perp \perp}$ and $r_{2} \in M_{2}^{\perp \perp}$. Since $\left\langle r_{1}, \alpha u_{i}+\alpha v_{i}\right\rangle=$ $\left\langle x, \alpha u_{i}+\alpha v_{i}\right\rangle=a_{i} \theta(\alpha)$ and likewise $\left\langle r_{2}, \alpha u_{i}-\alpha v_{i}\right\rangle=a_{i} \theta(\alpha)$ we have

$$
r_{1}=\sum_{i=1}^{\infty} \frac{a_{i}}{2} u_{i}+\frac{a_{i}}{2} v_{i}, \quad r_{2}=\sum_{i=1}^{\infty} \frac{a_{i}}{2} u_{i}-\frac{a_{i}}{2} v_{i} \text {. }
$$

By a similar expansion of $r_{1}$ and $r_{2}$ in terms of $\left\{u_{i}\right\}_{i=1}^{\infty}$ and $\left\{v_{i}\right\}_{i=1}^{\infty}$, we may write $r_{1}=w_{1}+w_{2}$ and $r_{2}=w_{1}-w_{2}$, where $w_{1}=\sum_{i=1}^{\infty}\left(a_{i} / 2\right) u_{i}$ and $w_{2}=$ $\sum_{i=1}^{\infty}\left(a_{i} / 2\right) v_{i}$. Clearly $\left\langle r_{1}, r_{1}\right\rangle=\left\langle r_{2}, r_{2}\right\rangle$. But $\left\langle r_{1}, r_{1}\right\rangle+\left\langle r_{2}, r_{2}\right\rangle=\langle x, x\rangle$. Thus $\left\langle r_{1}, r_{1}\right\rangle=2^{-1}\langle x, x\rangle$. Now $w_{1}=2^{-1} x$ so $\left\langle w_{1}, w_{1}\right\rangle=4^{-1}\langle x, x\rangle$. It follows that $\left\langle w_{2}, w_{2}\right\rangle=4^{-1}\langle x, x\rangle$. But $x^{\prime}=2 w_{2}$, hence $x^{\prime} \in V$ and $\left\langle x^{\prime}, x^{\prime}\right\rangle$ $=\langle x, x\rangle$.

5.6. Lemma. Let $(V,\langle\cdot, \cdot\rangle, \theta, D)$ be an infinite dimensional Hilbertian 
space satisfying $\mathrm{P}_{1}$ and $\mathrm{P}_{2}$. Let $\left\{u_{i}\right\}_{i=1}^{\infty}$ be an orthonormal sequence from $V$. Then if $x=\sum_{i=1}^{\infty} a_{i} u_{i} \in V$, we have $x^{\prime}=\sum_{i=1}^{\infty} \theta\left(a_{i}\right) u_{i} \in V$ and $\left\langle x^{\prime}, x^{\prime}\right\rangle=$ $\langle x, x\rangle$.

Proof. Without loss of generality we may assume $a_{i} \neq 0$ for all $i$ and that $\left\{v_{i}\right\}_{i=1}^{\infty}$ is an orthonormal sequence in $V$ which is orthonogonal to $\left\{u_{i}\right\}_{i=1}^{\infty}$. Let $\alpha \in D$ with $\alpha \theta(\alpha)=2^{-1}$ as in the proof of 5.5. Then for each $i$ define $\alpha_{i}=\theta(\alpha)$ and $\beta_{i}=\theta(\alpha) a_{i}^{-1} \theta\left(a_{i}\right)$. We have

$$
\alpha_{i} \theta\left(\alpha_{i}\right)=\theta(\alpha) \alpha=2^{-1}
$$

and

$$
\beta_{i} \theta\left(\beta_{i}\right)=\theta(\alpha) a_{i}^{-1} \theta\left(a_{i}\right) a_{i} \theta\left(a_{i}^{-1}\right) \alpha=2^{-1} .
$$

Thus the sequences $\left\{\alpha_{i} u_{i}+\beta_{i} v_{i}\right\}_{i=1}^{\infty}$ and $\left\{\alpha_{i} u_{i}-\beta_{i} v_{i}\right\}_{i=1}^{\infty}$ are mutually orthogonal and orthonormal and if they generate subspaces $M_{1}$ and $M_{2}$ of $V$ we may write $x=r_{1}+r_{2}, r_{1} \in M_{1}^{\perp \perp}$ and $r_{2} \in M_{2}^{\perp \perp}$. Here $\left\langle r_{1}, \alpha_{i} u_{i}+\beta_{i} v_{i}\right\rangle=$ $\left\langle x ; \alpha_{i} u_{i}+\beta_{i} v_{i}\right\rangle=a_{i} \alpha$ and we have

$$
r_{1}=\sum_{i=1}^{\infty} \frac{a_{i}}{2} u_{i}+\frac{\theta\left(a_{i}\right)}{2} v_{i}
$$

with a similar relation for $r_{2}$. Thus with $w_{1}=\sum_{i=1}^{\infty}\left(a_{i} / 2\right) u_{i}$ and $w_{2}=$ $\sum_{i=1}^{\infty}\left(\theta\left(a_{i}\right) / 2\right) v_{i}$, we may argue just as in the proof of 5.5 to see that if $y=2 w_{2}$ then $\langle y, y\rangle=\langle x, x\rangle$. The remainder of the proof follows from 5.5.

5.7. Lemma. Let $(V,\langle\cdot, \cdot\rangle, \theta, D)$ be an infinite dimensional Hilbertian space satisfying $P_{1}$ and $P_{2}$. Let $x=\sum_{i=1}^{\infty} a_{i} u_{i} \in V$ where $\left\{u_{i}\right\}_{i=1}^{\infty}$ is an orthonormal sequence from $V$. Then if $\left\{d_{i}\right\}_{i=1}^{\infty}$ is any sequence from $D$ with $d_{i} \theta\left(d_{i}\right)<$ $4^{-1}$ for each $i$ we have $y=\sum_{i=1}^{\infty} d_{i} a_{i} u_{i} \in V$ and $\langle y, y\rangle\langle\langle x, x\rangle$.

Proof. Again let $\alpha$ be an element with $\alpha \theta(\alpha)=2^{-1}$. Without loss of generality we may assume $\left\{v_{i}\right\}_{i=1}^{\infty}$ and $\left\{w_{i}\right\}_{i=1}^{\infty}$ are orthonormal sequences from $V$ which are mutually orthogonal and orthogonal to $\left\{u_{i}\right\}_{i=1}^{\infty}$. Now for each $i$ let $\alpha_{i}=\alpha$ and $\beta_{i}=\theta\left(\alpha^{-1}\right) \theta\left(d_{i}\right)$. Then

$$
\begin{aligned}
\beta_{i} \theta\left(\beta_{i}\right) & =\theta\left(\alpha^{-1}\right) \theta\left(d_{i}\right) d_{i} \alpha^{-1}=\theta\left(\alpha^{-1}\right) \alpha^{-1} \theta\left(d_{i}\right) d_{i} \\
& =(\alpha \theta(\alpha))^{-1} d_{i} \theta\left(d_{i}\right)<2^{-1} .
\end{aligned}
$$

Since $\alpha_{i} \theta\left(\alpha_{i}\right)=2^{-1}$ for each $i$ it follows that for each $i$ there is a $\gamma_{i}$ with

$$
\gamma_{i} \theta\left(\gamma_{i}\right)=1-\alpha_{i} \theta\left(\alpha_{i}\right)-\beta_{i} \theta\left(\beta_{i}\right) \text {. }
$$

Thus $\left\{\alpha_{i} u_{i}+\beta_{i} v_{i}+\gamma_{i} w_{i}\right\}_{i=1}^{\infty}$ is an orthonormal sequence in $V$. Let $M$ be the subspace of $V$ generated by this sequence and let $r=\sum_{i=1}^{\infty} \theta\left(a_{i}\right) u_{i}$. Then we may write $r=p+q, p \in M^{\perp \perp}$ and $q \in M^{\perp}$. A simple calculation of coefficients yields 


$$
p=\sum_{i=1}^{\infty}\left[\left(\theta\left(a_{i}\right) / 2\right) u_{i}+\theta\left(a_{i}\right) \theta\left(d_{i}\right) v_{i}+\theta\left(a_{i}\right) \theta(\alpha) \gamma_{i} w_{i}\right]
$$

If now $M_{1}$ is the subspace of $V$ generated by $\left\{v_{i}\right\}_{i=1}^{\infty}$ we may write $p=s+$ $t, s \in M_{1}^{\perp \perp}$ and $t \in M_{1}^{\perp}$. Here $s=\sum_{i=1}^{\infty} \theta\left(a_{i}\right) \theta\left(d_{i}\right) v_{i}$, and if we set

$$
z=\sum_{i=1}^{\infty} \theta\left(\theta\left(a_{i}\right) \theta\left(d_{i}\right)\right) v_{i}=\sum_{i=1}^{\infty} d_{i} a_{i} v_{i}
$$

we have by 5.5 and 5.6,y $\in V$ and

$$
\langle y, y\rangle=\langle z, z\rangle=\langle s, s\rangle\langle\langle p, p\rangle\langle\langle r, r\rangle=\langle x, x\rangle .
$$

We are now ready to prove our main theorem.

5.8. TheOREM. Let $(V,\langle\cdot, \cdot\rangle, \theta, D)$ be an infinite dimensional Hilbertian space satisfying $\mathrm{P}_{1}$ and $\mathrm{P}_{2}$. Then one of the following three cases holds:

(i) $D=\mathbf{R}$, the real field, and $\theta=$ identity;

(ii) $D=\mathbf{R}(i)$, the complex field, and $\theta$ is the usual conjugation;

(iii) $D=\mathbf{R}(i, j)$, the quaternions, and $\theta$ is the usual conjugation.

As a consequence, $V$ with the form $\langle\cdot, \cdot\rangle$ is one of the classical Hilbert spaces over the reals, the complexes, or the quaternions.

Proof. The crucial step here is to show that $F$ is Archimedean, hence let us suppose it is not. By application of $P_{1}$ we may find an orthonormal sequence $\left\{u_{i}\right\}_{i=1}^{\infty}$ in $V$. Let $\left\{a_{i}\right\}_{i=1}^{\infty}$ be a sequence of positive rational numbers in $F$ such that $\sum_{i=1}^{\infty} a_{i}^{2}=3^{-1}$. Then applying 5.3 with $A$ equal to the subfield of $F$ consisting of the rationals we obtain an element $x=\sum_{i=1}^{\infty} b_{i} u_{i} \in V$ with $\langle x, x\rangle\left\langle 2^{-1}\right.$ and such that $\left(a_{i}-b_{i}\right) \theta\left(a_{i}-b_{i}\right)$ is infinitesimal for each $i$. Knowing the action of $\theta$ and form of elements by 4.2 it is clear that each $b_{i} \theta\left(b_{i}\right)$ is a finite positive element of $F$. Now let $f$ be some nonzero infinitesimal element from $F$. Then with $d_{i}^{\prime}=f b_{i}^{-1}$ for each $i$ we have

$$
\begin{aligned}
d_{i}^{\prime} \theta\left(d_{i}^{\prime}\right) & =f b_{i}^{-1} \theta\left(b_{i}^{-1}\right) \theta(f)=f^{2}\left(\theta\left(b_{i}\right) b_{i}\right)^{-1} \\
& =f^{2}\left(b_{i} \theta\left(b_{i}\right)\right)^{-1}<4^{-1} .
\end{aligned}
$$

It follows by 5.7 that

$$
y^{\prime}=\sum_{i=1}^{\infty} f u_{i} \in V
$$

Thus

$$
z=f^{-1} y^{\prime}=\sum_{i=1}^{\infty} u_{i} \in V .
$$

Now if $z_{n}$ represents the projection of $z$ onto the subspace generated by $\left\{u_{i}\right\}_{i=1}^{n}$ we have 


$$
n=\sum_{i=1}^{n}\left\langle u_{i}, u_{i}\right\rangle=\left\langle z_{n}, z_{n}\right\rangle\langle\langle z, z\rangle .
$$

It follows that $\langle z, z\rangle=h$ is infinite. Now let $c \in D$ with $c \theta(c)=h^{-1}$. For each $i$ let $d_{i}=c b_{i}^{-1}$. Then

$$
d_{i} \theta\left(d_{i}\right)=c b_{i}^{-1} \theta\left(b_{i}^{-1}\right) \theta(c)=c \theta(c)\left(b_{i} \theta\left(b_{i}\right)\right)^{-1} \leqslant 4^{-1}
$$

because $h^{-1}$ is infinitesimal. By 5.7 with $y=\sum_{i=1}^{\infty} d_{i} b_{i} u_{i}$ we have $\langle y, y\rangle \leqslant$ $\langle x, x\rangle\left\langle 2^{-1}\right.$. However $y=c z$ and this demands $\langle y, y\rangle=1$. From this contradiction we conclude that $F$ is Archimedean.

The remainder of the proof is now simple. First from 5.4 it follows that $F=\mathbf{R}$, the real field. With this result (i)-(iii) are an almost trivial consequence of (i)-(iii) of 4.2. It is only necessary to note that in case (ii) of 4.2 if $F=\mathbf{R}$ then there is an $a \in F$ such that with $i=a I, i^{2}=-1$ and likewise for case (iii). We conclude that $V$ with the form $\langle\cdot, \cdot\rangle$ is a pre-Hilbert space over the reals, the complexes, or the quaternions. It then follows from a result due to Piron and to Araki $[15,7.42]$ that $V$ is complete and thus a Hilbert space. This completes the proof of the theorem.

5.9. Corollary. The only infinite dimensional complete projective logics which are probabilistic are the classical logics of the real, complex, or quaternionic Hilbert spaces.

5.10. COROLLARY. The only infinite dimensional complete projective logics over an algebraically closed field are the classical logics of the complex Hilbert spaces. Likewise the only infinite dimensional complete projective logics over a real closed field are the classical logics of the real Hilbert spaces.

Here 5.10 is a consequence of the fact that in an algebraically closed or real closed field the condition $\mathrm{N}$ is satisfied regardless of the choice of the involutive antiautomorphism $\theta$. This result is of interest in its relation to a

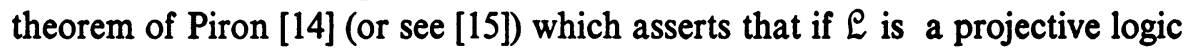
of dimension $\kappa_{0}$ over the complex field and if the corresponding involutive antiautomorphism $\theta$ is continuous (continuity need not be assumed in the real or quaternionic cases) then $\mathcal{E}$ is just the logic of separable complex Hilbert space. Result 5.10 shows that in fact continuity need not be assumed in the complex case. Briefly, it is always possible to induce a new topology on the complex field using the original topology and a discontinuous field automorphism in such a way that $\theta$ is continuous. An example of an infinite dimensional logic over the complex field which illustrates these possibilities is given in $[17, \S 7]$.

6. Axioms for the standard logic of separable complex Hilbert space. By employing the results of the previous section we are enabled to give here a 
strictly lattice-theoretic characterization of the lattice of closed subspaces of separable complex Hilbert space. To begin we shall assume $\mathcal{L}$ is a logic and we shall proceed by setting down a system of axioms which single out the standard logic of complex Hilbert space from the class of all logics.

We shall need a rather general definition of dimension in a lattice. Let $S$ be a subset of $\mathcal{L}$ and let $x$ be an element in $\mathcal{L}$. If $t<x$ for each $t \in S$ we shall say $x$ bounds $S$. Then following [2] we define the dimension of $x$ or $d[x]$ to be the least cardinal which is an upper bound for all the cardinals of subsets $S$ of $\mathcal{E}$ which do not contain zero, are chains, and are bounded by $x$. We further set $d[\mathcal{L}]=d[1]$. According to this definition the points of $\mathcal{L}$ are just the elements of dimension 1.

We first list the axioms and then discuss their sufficiency.

Axioms I. $\mathcal{L}$ has at least one point.

AxIom II. $d[\ell]=\kappa_{0}$.

Axiom III. If $a$ and $a^{\perp}$ are nonzero elements of $\mathcal{L}$ and $x$ is a point in $\mathcal{L}$, then there are points $y$ and $z$ in $\mathcal{L}$ with $y<a, z<a^{\perp}$ and $x<y \vee z$.

Axiom IV. If $a, b$ and $c$ are elements of $\mathcal{L}$ and if $a \vee b$ is less than the sum of a finite set of points in $\mathcal{L}$, and $c<a$, then $a \wedge(b \vee c)=(a \wedge b) \vee c$.

Axiom V. If $x$ and $y$ are distinct points in $\mathcal{E}$ then there is a distinct third

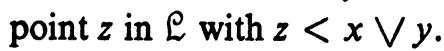

Axıom VI. Given any four distinct points $u, v, w, x$ with $u \vee v=w \vee x$ there exist points $y$ and $z$ in $\mathcal{E}$ with $y<(v \vee z), y<(w \vee x), z<(w \vee x), z$ $<(u \vee y)$, and such that $d[w \vee x \vee y \vee z]=2$ and

$$
(u \vee y) \wedge(z \vee x)<[(v \vee z) \wedge(w \vee y)] \vee[(u \vee v) \wedge(z \vee y)]
$$

Axıom VII. If $u, v, w, x, y$ and $z$ are six distinct points in $\mathcal{E}$ with $d[u \vee v$ $\vee w \vee x \vee y \vee z]=2$ and if $(u \vee v) \wedge(w \vee z) \wedge(y \vee z) \neq 0$ and $(x \vee$ y) $\wedge(z \vee u) \wedge(v \vee w) \neq 0$, then $(u \vee x) \wedge(v \vee y) \wedge(w \vee z) \neq 0$

Our first step in demonstrating the sufficiency of these axioms will be to show that they imply $\mathcal{L}$ is a complete projective logic.

6.1. LeMMA. Any logic $\mathcal{L}$ satisfying Axioms I-V is a complete projective logic.

Proof. The completeness follows from Axiom II and projectivity [15, $\mathrm{p}$. 183] hence it is enough to establish that $\mathcal{L}$ is projective. Let $a$ be a nonzero

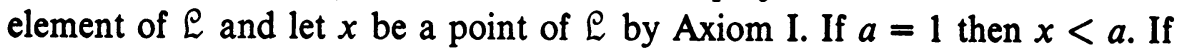
$a \neq 1$ then $a^{\perp} \neq 0$ and by Axiom III and the existence of $x$ there is a point less than $a$. Thus condition (i) of projectivity (see \$2) holds. For condition (ii) let $a$ be the sum of a nonempty finite set of points in $\mathcal{E}$. By the definition of a logic and by Axiom IV it is clear that $\mathcal{L}[0, a]$ is a complemented modular lattice of finite dimension. It is now a consequence of Axiom $V$ and [15, 2.15] that $\mathcal{L}[0, a]$ is a geometry and condition (ii) is satisfied. Condition (iii) is just Axiom III while condition (iv) is a simple consequence of conditions (i) and 
(ii), Axiom II and the definition of a logic.

It is now clear that if $\mathcal{L}$ satisfies Axioms $I-V$ then $\mathcal{L}$ is represented by a Hilbertian space $(V,\langle\cdot, \cdot\rangle, \theta, D)$.

6.2. Lemma. The space $(V,\langle\cdot, \cdot\rangle, \theta, D)$ is a Hilbertian space representing a logic $\mathcal{L}$ which satisfies Axiom VI if and only if every element of $D$ has a square root in $D$.

Proof. Let $(V,\langle\cdot, \cdot\rangle, \theta, D)$ be a Hilbertian space representing a logic $\mathfrak{L}$ which satisfies Axiom VI. Now let $u, v$, and $w$ be distinct points on a line $c$ in $\mathcal{L}$ and let $a$ be some plane in $\mathcal{E}$ containing $c$. Then we recall [15] that the multiplicative group $D^{\prime}=D-\{0\}$ may be represented as the group $M(c ; u, v)$ consisting of the identity and the general projectivities in $\mathrm{P}_{2}(c)$ as determined in $\mathcal{E}[0, a]$ and with $u$ and $v$ as their first and second canonical fixed points, respectively. Further the elements of $M(c ; u, v)$ are uniquely determined by their action on $w$ and may be represented as $\left\{q_{x}: x<c, x \neq\right.$ $u$ and $x \neq v$ \} where $q_{x}(w)=x$. Thus we must establish that for any such $x$ there is a $\gamma$ with $q_{\gamma} \circ q_{\gamma}=q_{x}$. If $x=w$ we may set $\gamma=w$ for a solution so let us assume $x \neq w$. Then by Axiom VI there are points $y$ and $z$ in $\mathcal{E}$ with prescribed properties in relation to the points $u, v, w$, and $x$. Let us assume for the moment that $y$ and $z$ are in $\mathcal{L}[0, a]$. Then with $\alpha=(v \vee z) \wedge(w \vee y)$, $\beta=(u \vee y) \wedge(z \vee x)$ and $\gamma=(u \vee v) \wedge(z \vee y)$, Axiom VI asserts that the figure is correct and $\alpha, \beta$ and $\gamma$ are collinear.

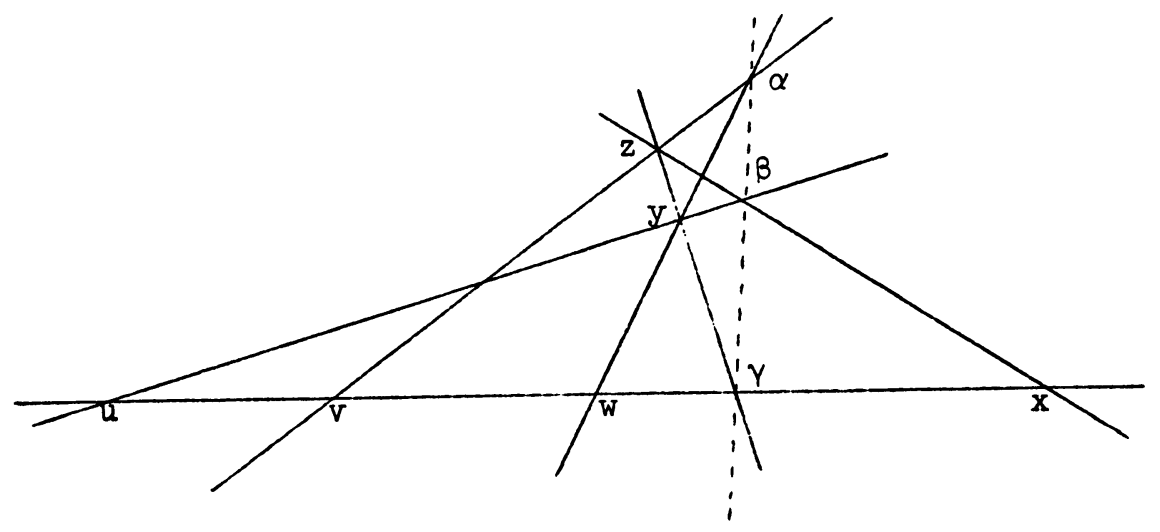

It can then be easily seen from the figure using $y$ and $\beta$ as centers of perspectivity that $q_{\gamma} \circ q_{\gamma}=q_{x}$. Now if $y$ and $z$ are not in $\mathcal{E}[0, a]$ then there is some other plane $b$ containing $y, z$, and $c$. But then by $[15,5.3]$ there is an isomorphism between $\mathcal{L}[0, a]$ and $\mathcal{L}[0, b]$ which leaves $\mathcal{E}[0, c]$ fixed and hence corresponding to $y$ and $z$ there are points $y^{\prime}$ and $z^{\prime}$ in $\mathcal{L}[0, a]$ which satisfy all the conditions of Axiom VI with respect to $u, v, w$, and $x$. Thus in any case 
there is a $q_{\gamma}$ with $q_{\gamma} \circ q_{\gamma}=q_{x}$. This establishes the only if part of the lemma. The if part is trivial.

6.3. TheOREM. A logic $\mathcal{L}$ satisfies Axioms I-VII if and only if it is isomorphic to the standard logic of separable complex Hilbert space.

Proof. Suppose $\mathcal{L}$ satisfies Axioms I-VII. Then by $6.1, \mathcal{L}$ may be represented by a Hilbertian space $(V,\langle\cdot, \cdot\rangle, \theta, D)$. By 6.2 every element of $D$ has a square root in $D$ and by Axiom VII, which is Pappus' theorem, $D$ is a field. Thus $(V,\langle\cdot, \cdot\rangle, \theta, D)$ satisfies condition $\mathrm{N}$ and since $\mathcal{L}$ is infinite dimensional it must by 5.9 be isomorphic to one of the classical logics of real, complex, or quaternionic Hilbert space. Since the reals do not have a square root for every element and the quaternions are not commutative we are left with the complex field. As Axiom II sets the dimension at $\kappa_{0}$ we conclude that $\mathcal{E}$ is isomorphic to the logic of separable complex Hilbert space. The converse statement that the logic of separable complex Hilbert space satisfies Axioms I-VII follows easily from [15, 7.40], the result 6.2 , and the fact that commutativity implies Pappus' theorem.

It is well known that one cannot hope to arrive at the complex field by purely algebraic hypotheses. In the case of the finite dimensional geometries, assumptions concerning the character of the topology which may be defined on the geometry have proved useful in singling out the geometries associated with the real, complex, or quaternionic numbers [10], [16], [18]. In our case, Axiom II and the countable completeness inherent in the definition of a logic supply the essential nonalgebraic character to arrive at the complex field .

\section{BIBLIOGRAPHY}

1. E. G. Beltrametti and G. Cassinelli, Quantum mechanics and p-adic numbers, Found. Phys. 2 (1972), 1-7. MR 48 \#10328.

2. G. Birkhoff, Lattice theory, rev. ed., Amer. Math. Soc. Colloq. Publ., vol. 25, Amer. Math. Soc., Providence, R.I., 1948. MR 10, 673.

3. G. Birkhoff and J. von Neumann, The logic of quantum mechanics, Ann. of Math. (2) 37 (1936), 823-843.

4. P. C. Deliyannis, Vector space models of abstract quantum logics, J. Mathematical Phys. 14 (1973), 249-253. MR 48 \#3401.

5. J.-P. Eckmann and $\mathrm{Ph}$. Ch. Zabey, Impossibility of quantum mechanics in a Hilbert space over a finite field, Helv. Phys. Acta 42 (1969), 420-424. MR 39 \# 7904.

6. A. M. Gleason, Measures on the closed subspaces of a Hilbert space, J. Math. Mech. 6 (1957), 885-893. MR 20 \#2609.

7. S. P. Gudder and C. Piron, Observables and the field in quantum mechanics J. Mathematical Phys. 12 (1971), 1583-1588. MR 46 \#8552.

8. S. P. Gudder and P. Stanley, Quantum logics, physical space, position observables and symmetry, Rep. Mathematical Phys. 4 (1973), 193-202. MR 48 \#3379.

9. S. S. Holland, Jr., Remarks on type I Baer and Baer *-rings, J. Algebra 27 (1973), 516-522. MR 48 \#8554. 
10. A. N. Kolmogorov, Zur Begründung der Projectiven Geometrie, Ann. of Math. (2) 33 (1932), 175-176.

11. M. J. Maczyniski, Hilbert space formalism of quantum mechanics without the Hilbert space axiom, Rep. Mathematical Phys. 3 (1972), no. 3, 209-219. MR 47 \#9982.

12. The field of real numbers in axiomatic quantum mechanics, J. Mathematical Phys. 14 (1973), 1469-1471. MR 48 \#7798.

13. R. P. Morash, The orthomodular identity and metric completeness of the coordinatizing division ring, Proc. Amer. Math. Soc. 27 (1971), 446-448; Errata, ibid. 29 (1971), 627. MR 42 \# 7570; 43 \#2016.

14. C. Piron, Axiomatic quantique, Thése, Univ. de Lausanne, Faculté des Sciences; Birkhäuser, Basel, 1964, pp. 439-468.

15. V. S. Varadarajan, Geometry of quantum theory, Vol. I, Van Nostrand, Princeton, N.J., 1968.

16. E. Weiss and N. Zierler, Locally compact division rings, Pacific J. Math. 8 (1958), 369-371. MR 22 \# 12170.

17. W. J. Wilbur, Quantum logic and the locally convex spaces, Trans. Amer. Math. Soc. 207 (1975), 343-360. MR 51 \#3849.

18. N. Zierler, Axioms for non-relativistic quantum mechanics, Pacific J. Math. 11 (1961), 1151-1169. MR 25 \# 4385.

19. On the lattice of closed subspaces of Hilbert space, Pacific J. Math. 19 (1960), 583-586. MR 34 \#2509.

Department of Mathematics, ANDrews University, BerRien Springs, Michigan 49104

Current address: 11209 Rosarita Drive, Loma Linda, California 92354 